\title{
The Correlation of the Neutrophil-Lymphocyte Ratio and the Platelet-Lymphocyte Ratio With Pathological Findings in Neuroendocrine Tumors
}

\author{
Ozgur Kulahci ${ }^{1}$, Tolga Koseci ${ }^{2}$ \\ 1. Department of Pathology, University of Health Sciences, Adana City Education and Research Hospital, Adana, TUR \\ 2. Department of Medical Oncology, University of Health Sciences, Adana City Education and Research Hospital, \\ Adana, TUR
}

Corresponding author: Ozgur Kulahci, ozgurkulahci@hotmail.com

\section{Abstract}

\section{Introduction}

The relationship between clinical prognostic factors and blood neutrophil-lymphocyte ratio (NLR) and platelet-lymphocyte ratio (PLR) in some tumors has been investigated. In this study, we examined whether there is a relationship between pathological prognostic factors and NLR as well as PLR only in neuroendocrine tumors (NETs).

\section{Methods}

A total of 115 patients with a NET diagnosis between 2014-2020 were included in the study. The efficiency of NLR and PLR in predicting distant metastases was determined by analyzing the receiver operating characteristic (ROC) curve. The relationship between histopathological parameters was also compared.

\section{Results}

The cut-off value of NLR was 3.01 for predicting distant metastasis. At this value, the specificity was $73.7 \%$, the sensitivity was $70.7 \%$, and the likelihood ratio was 2.51 . There was a significant relationship between NLR and tumor localization, histological grade, mitosis, Ki-67, distant metastasis, and lymphovascular invasion (all $\mathrm{p}<0.001$ ). The cut-off value of the PLR in predicting distant metastasis was 134.4. At this value, the specificity was $59.6 \%$, the sensitivity was $58.6 \%$, and the likelihood ratio was 1.44 . There was no significant relationship between PLR and the histopathological findings (all $\mathrm{p}>0.05$ ).

\section{Conclusions}

In our study, a high histological grade, high mitosis, a high Ki-67 proliferation index, distant metastasis, and lymphovascular invasion were found in patients with NLR at a cut-off value above 3.01. However, we could not attain the same results for PLR. For trucut and endoscopic biopsies in particular, follow-up of patients with grades 1 and 2 NETs along with histopathological findings and evaluation of NLR in peripheral blood may be useful. NLR, which is an easily accessible inflammatory marker, can be used as an independent predictive factor in NETs.

Review began 07/30/2021 Review ended 08/03/2021 Published 08/13/2021

(c) Copyright 2021 Kulahci et al. This is an open access article distributed under the terms of the Creative Commons Attribution License CC-BY 4.0., which permits unrestricted use, distribution, and reproduction in any medium, provided the original author and source are credited.
Categories: Pathology, Oncology, Hematology

Keywords: neuroendocrine tumours, pathology, neutrophils, lymphocytes, tumor grade

\section{Introduction}

Neuroendocrine tumors (NETs) originate from neuroendocrine cells with characteristic histopathological and immunohistochemical properties [1]. NETs can appear in different parts of the body, including the gastrointestinal system, respiratory system, central nervous system, larynx, thyroid, breasts, skin, and urogenital system [2]. They most commonly occur in the gastrointestinal tract, lungs, and pancreas. It is thought that a systemic inflammatory response is associated with poor prognosis in many cancers in terms of predicting tumor invasion, metastasis, and angiogenesis [3]. Recently, the blood neutrophil-lymphocyte ratio (NLR) and the platelet-lymphocyte ratio (PLR) have been investigated in relation to poor prognosis in various oncological tumors $[4,5]$. Because they can be easily measured in cancer patients, NLR and PLR in peripheral blood have been examined in predicting prognostic results and risk classification before treatment. A high NLR has been associated with advanced stage and poor prognosis in many tumor types, such as stomach and breast cancers [6,7]. In patients with thrombocytosis, a connection has been established between poor prognosis and shorter survival in various tumor types, such as lung, colon, gastric, breast, and ovarian cancers [8]. Various studies have been conducted involving NLR and PLR regarding prognosis in NETs. A relationship was determined between a high NLR and large tumor size, an advanced stage, a high grade, as well as decreased survival time in NETs [9]. In another study, the preoperative NLR was determined as a potential independent marker for lymph node metastasis and recurrence-free survival 
[10]. While there are studies indicating an increase in NLR and PLR as the grade increases in NETs, some other studies have indicated no relationship between the increase in PLR and metastasis [11,12]. Studies showing the relationship between NLR, PLR, and NETs are generally related to clinical parameters and survival. This study was planned based on histopathological parameters. The aim was to determine the relationship between NLR and PLR in the peripheral blood and pathological prognostic factors in NETs. The cut-off values were determined for predicting distant metastasis by analyzing the receiver operating characteristic (ROC) curve for NLR and PLR. Its relationship with histopathological parameters was also evaluated.

\section{Materials And Methods \\ Pathological evaluation}

A total of 115 patients with NETS who were seen between 2014-2020 in our hospital's pathology department were retrospectively analyzed. Approval for the study was obtained from the University of Health Sciences, Adana City Education and Research Hospital Ethics Committee (Decision no: 957). Patients who were diagnosed with NET via tru-cut biopsy, endoscopic biopsy, colonoscopic biopsy, excisional biopsy, or resection materials were re-evaluated by an experienced pathologist. For cases of NETs with metastases on imaging, those with a diagnosis of metastasis that was confirmed by biopsy or tumor resection were included in the study. Patients who were not diagnosed with a biopsy, those who had a recurrent tumor and received chemoradiotherapy were excluded from the study. Age, gender, tumor localization, histological grade, mitosis, distant metastasis, lymphovascular invasion, perineural invasion, immunohistochemical Ki-67, synaptophysin and chromogranin A staining, and a correlation between NLR and PLR in peripheral blood were evaluated in all patients. Tumor localizations were grouped into those in the gastroenteropancreatic system (esophagus, stomach, small intestine, large intestine, appendix, pancreas), lungs, and other organs.

The histological grade of NET was evaluated according to the 2019 World Health Organization (WHO) classification of digestive system tumors and the 2015 WHO classification of lungs, pleura, thymus, and heart tumors. For histological grading of the gastroenteropancreatic system and other localizations, it was grouped either as a grade 1 well-differentiated NET, a grade 2 well-differentiated NET, or a grade 3 welldifferentiated NET [13]. Typical carcinoid tumors were considered as grade 1 in the lung; atypical carcinoid tumors were considered as grade 2 , and neuroendocrine carcinomas were considered as grade 3 [14]. According to the WHO classification, mitosis was grouped as less than 2 in $2 \mathrm{~mm}^{2}$, between 2-20, and more than 20 upon microscopic examination for the gastroenteropancreatic system and other localizations [13]. For the lung, it was grouped as less than 2 in $2 \mathrm{~mm}^{2}$, between 2-10, and more than 10 [14]. If the biopsy showed less than $2 \mathrm{~mm}^{2}$, the entire tissue was evaluated. Immunohistochemical stains were completed with Bond-Max fully automated IHC and ISH (Leica Biosystems Division of Leica Microsystems Inc, Buffalo Grove, IL) according to the manufacturer's staining procedure. Ki-67 (Novocastra ${ }^{\mathrm{TM}}$ Liquid Mouse Monoclonal Antibody Ki-67 Antigen, Leica Biosystems Newcastle Ltd, Newcastle Upon Tyne, UK), which was used for the gastroenteropancreatic system and other localizations, was grouped as less than $3 \%$ upon microscopic examination, between $3-20 \%$, and greater than $20 \%$. It was grouped at up to $5 \%$, between 5 $20 \%$, and greater than $20 \%$ for the lung. Ki-67-stained slides were counted manually with images taken with the camera and then printed out in color. Each tumor slide was manually scanned with a microscope at x10 objective. The largest Ki-67 positivity (hot spot) area was chosen for photography and printing. The color image of the hot spot was captured with the camera snapshot. It was printed on white paper. Ki-67-positive dark brown tumor nuclei were counted and the Ki-67 proliferation index was calculated. Light brown or pale staining nuclei were ignored during counting. Distant metastasis, lymphovascular invasion, perineural invasion, synaptophysin (NCL-L-SYNAP-299, Leica Biosystems Newcastle Ltd), and chromogranin A (NCLL-CHROM-430, Leica Biosystems Newcastle Ltd) were grouped as negative and positive. The blood values of patients taken from the peripheral vein within two weeks before biopsy or surgery were analyzed with a Beckman Coulter DXH-800 (Beckman Coulter Eurocenter, Nyon, Switzerland). Neutrophil, platelet, and lymphocyte counts were determined. NLR was calculated by dividing the neutrophil count by the lymphocyte count. PLR was calculated by dividing the platelet count by the lymphocyte count. An ROC curve analysis was performed with NLR and PLR to determine the relationship between cut-off values, and the above pathological parameters for predicting metastasis were compared.

\section{Statistical analysis}

SPSS Statistics version 19 was used for statistical evaluation of the data obtained in the study (IBM, Armonk, NY). Descriptive statistics related to variables such as age, NLR, and PLR were presented as the median (range). Categorical variables such as gender, tumor localization, mitosis, Ki-67 index, histological grade, distant metastasis, lymphovascular invasion, perineural invasion, and synaptophysin and chromogranin A staining were presented in numbers (n) and percentages (\%). An ROC curve analysis was performed to determine the cut-off value in predicting metastasis of NLR and PLR. The area values under the curve that were obtained from the ROC curve analysis were used to compare predictive activities of NLR and PLR. Categorical variables were analyzed using the Chi-squared test, Fisher's exact test, or likelihood ratio test. Sensitivity, specificity, and likelihood ratio parameters were used to investigate the accuracy of diagnostic tests for distant metastasis with NLR and PLR. A p-value $<0.05$ was considered statistically significant. 


\section{Cureus}

\section{Results}

All included patients were between the ages of 11 and 88 years; two cases were under the age of 18 , and their tumors were located in the appendix. While $47.8 \%$ of the patients were female, $52.2 \%$ were male, and $56.5 \%$ of the tumors were localized in the gastroenteropancreatic system. Distant metastases were present in $49.6 \%$ of the patients. Categorical variables were analyzed using the Chi-squared test, Fisher's exact test, or likelihood ratio test. The clinical and pathological features of NET patients are shown in Table 1.

\section{Variable}

Age in years, median (range)

Male gender

Localization

\section{GEP system}

Lung

Other

Histological grade (G)

G1

G2

G3

Mitosis*

$<2$

$2-20(2-10)$

$>20$ (>10)

$\mathrm{Ki}-67^{*}$

$<3(5) \%$

$3-20(5-20) \%$

$>20 \%$

Metastasis

Negative

Positive

LVI

Negative

Positive

PNI

Negative

Positive

Synaptophysin

Negative

Positive

Chromogranin A

Negative

Positive

NLR, median (range)
$58(50.4)$

47 (40.9)

68 (59.1)

92 (80)

$23(20)$

113 (98.3)

12 (10.4)

All patients, $\mathbf{n}(\%)$

$61(11-88)$

60 (52.2)

$65(56.5)$

35 (30.4)

15 (13)

$44(38.3)$

12 (10.4)

59 (51.3)

$44(38.3)$

12 (10.4)

59 (51.3)

$44(38.3)$

12 (10.4)

59 (51.3)

57 (49.6)

103 (89.6)

3.04 (0.73-52.61) 


\section{Cureus}

\section{TABLE 1: Clinical and pathological features of patients with NET}

*Values in parentheses for mitosis and Ki-67 indicate the lungs

NET: neuroendocrine tumor; GEP: gastroenteropancreatic; LVI: lymphovascular invasion; PNI: perineural invasion; NLR: neutrophil-lymphocyte ratio; PLR: platelet-lymphocyte ratio

An ROC curve analysis was performed for NLR and PLR. The area under the curve in NLR was 75.6\%, and the confidence interval was $66.7-84.6 \%$. The cut-off value of NLR was 3.01 in predicting distant metastasis. At this value, the specificity was $73.7 \%$, the sensitivity was $70.7 \%$, and the likelihood ratio was 2.51 . The area under the curve in the PLR was 63.1\%, and the confidence interval was 52.4-73.7\%. The cut-off value of the PLR for predicting distant metastasis was 134.4 . At this value, the specificity was $59.6 \%$, the sensitivity was $58.6 \%$, and the likelihood ratio was 1.44 .

A correlation was found between the cut-off value of NLR and tumor localization, histological grade, mitosis, Ki-67 proliferation index, metastasis, and lymphovascular invasion (all p<0.001). A higher histological grade, high mitosis, and a high Ki-67 proliferation index were determined in the group with an NLR cut-off value above 3.01. This group was mostly located in the lungs and other tissues, and it was more in distant metastasis and lymphovascular invasion. There was no correlation between NLR cut-off value and perineural invasion and synaptophysin and chromogranin A immunohistochemical staining (all p>0.05). The correlation between NLR and categorical variables is shown in Table 2. 


\section{Cureus}

\begin{tabular}{|c|c|c|c|}
\hline & NLR <3.01, n (\%) & NLR >3.01, n (\%) & P-value \\
\hline Localization & & & $<0.001$ \\
\hline GEP system & $42(64.6)$ & $23(35.4)$ & \\
\hline Lung & $13(37.1)$ & $22(62.9)$ & \\
\hline Other & $2(13.3)$ & $13(86.7)$ & \\
\hline Histological grade (G) & & & $<0.001$ \\
\hline G1 & $36(81.8)$ & $8(18.2)$ & \\
\hline G2 & $3(25)$ & $9(75)$ & \\
\hline G3 & $18(30.5)$ & $41(69.5)$ & \\
\hline Mitosis` & & & $<0.001$ \\
\hline$<2$ & $36(81.8)$ & $8(18.2)$ & \\
\hline $2-20(2-10)$ & $3(25)$ & $9(/ 5)$ & \\
\hline$>20(>10)$ & $18(30.5)$ & $41(69.5)$ & \\
\hline Ki-67* & & & $<0.001$ \\
\hline$<3(5) \%$ & $36(81.8)$ & $8(18.2)$ & \\
\hline $3-20(5-20) \%$ & $3(25)$ & $9(75)$ & \\
\hline$>20 \%$ & $18(30.5)$ & $41(69.5)$ & \\
\hline Metastasis & & & $<0.001$ \\
\hline Negative & $42(12.4)$ & $16(27.6)$ & \\
\hline Positive & $15(26.3)$ & $42(73.7)$ & \\
\hline LVI & & & $<0.001$ \\
\hline Negative & $37(78.7)$ & $10(21.3)$ & \\
\hline Positive & $20(29.4)$ & $48(70.6)$ & \\
\hline PNI & & & 0.113 \\
\hline Negative & $49(53.3)$ & $43(46.7)$ & \\
\hline Positive & $8(34.8)$ & $15(65.2)$ & \\
\hline Synaptophysin & & & 0,99 \\
\hline Negative & $1(50)$ & $1(50)$ & \\
\hline Positive & $56(49.6)$ & $57(50.4)$ & \\
\hline Chromogranin A & & & 0.235 \\
\hline Negative & $4(33.3)$ & $8(66.7)$ & \\
\hline Positive & $53(51.5)$ & $50(48.5)$ & \\
\hline
\end{tabular}

TABLE 2: Correlation between NLR in peripheral blood and histopathological findings

*Values in parentheses for mitosis and $\mathrm{Ki}-67$ indicate the lungs

GEP: gastroenteropancreatic; LVI: lymphovascular invasion; PNI: perineural invasion; NLR: neutrophil-lymphocyte ratio

No relation was determined between the cut-off value of PLR and tumor localization, histological grade, mitosis, Ki-67 proliferation index, metastasis, lymphovascular invasion, perineural invasion, synaptophysin, and chromogranin A immunohistochemical staining (all p>0.05). The correlation between PLR and the 


\section{Cureus}

categorical variables is summarized in Table 3.

\begin{tabular}{|c|c|c|c|}
\hline & PLR <134.4, n (\%) & PLR >134.4, n (\%) & P-value \\
\hline Localization & & & 0.217 \\
\hline GEP system & $36(55.4)$ & $29(44.6)$ & \\
\hline Lung & $15(42.9)$ & $20(57.1)$ & \\
\hline Other & $5(33.3)$ & $10(66.7)$ & \\
\hline Histological grade (G) & & & 0.086 \\
\hline G1 & $27(61.4)$ & $17(38.6)$ & \\
\hline G2 & $4(33.3)$ & $8(66.7)$ & \\
\hline G3 & $25(42.4)$ & 34 (57.6) & \\
\hline Mitosis* & & & 0.086 \\
\hline$<2$ & $27(61.4)$ & 17 (38.6) & \\
\hline $2-20(2-10)$ & $4(33.3)$ & $8(66.7)$ & \\
\hline$>20(>10)$ & $25(42.4)$ & $34(57.6)$ & \\
\hline Ki-67* & & & 0.086 \\
\hline$<3(5) \%$ & $27(61.4)$ & $17(38.6)$ & \\
\hline 3-20 (5-20)\% & $4(33.3)$ & $8(66.7)$ & \\
\hline$>20 \%$ & $25(42.4)$ & 34 (57.6) & \\
\hline Metastasis & & & 0.076 \\
\hline Negative & 33 (59.6) & $25(43.1)$ & \\
\hline Positive & $23(40.4)$ & $34(59.6)$ & \\
\hline LVI & & & 0.119 \\
\hline Negative & $27(57.4)$ & 20 (42.6) & \\
\hline Positive & $29(42.6)$ & $39(57.4)$ & \\
\hline PNI & & & 0.926 \\
\hline Negative & 45 (48.9) & $47(51.1)$ & \\
\hline Positive & $11(47.8)$ & $12(52.2)$ & \\
\hline Synaptophysin & & & 0.165 \\
\hline Negative & 0 & $2(100)$ & \\
\hline Positive & 56 (49.6) & $57(50.4)$ & \\
\hline Chromogranin A & & & 0.607 \\
\hline Negative & $5(41.7)$ & $7(58.3)$ & \\
\hline Positive & $51(49.5)$ & $52(50.5)$ & \\
\hline
\end{tabular}

\section{TABLE 3: Correlation between PLR in peripheral blood and histopathological findings}

*Values in parentheses for mitosis and Ki-67 indicate the lungs

GEP: gastroenteropancreatic; LVI: lymphovascular invasion; PNI: perineural invasion; PLR: platelet-lymphocyte ratio 


\section{Cureus}

grade 1, 2, and 3 NETs are shown in Figure 1.
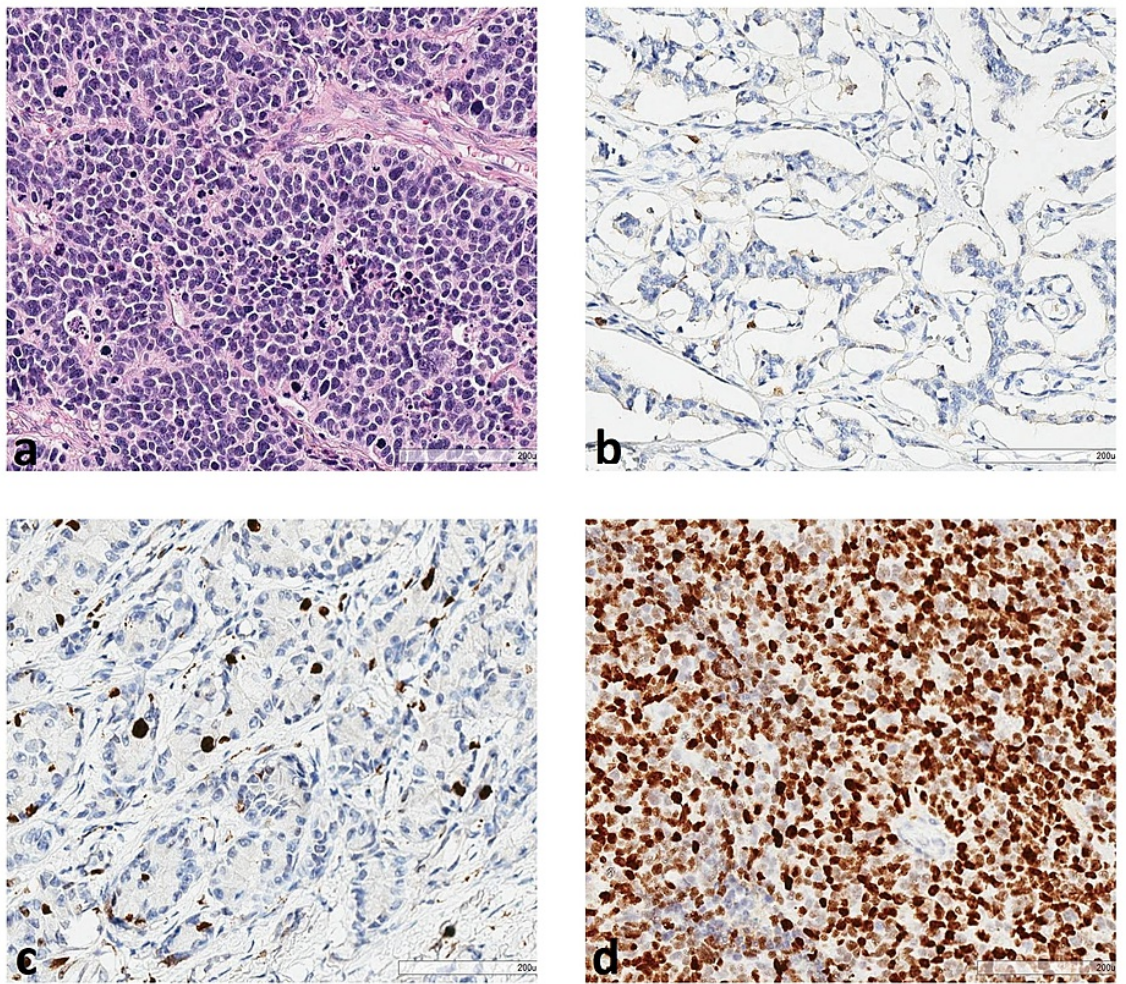

FIGURE 1: (a) Grade 3 neuroendocrine carcinoma (hematoxylin and eosin 200x); (b) Grade 1 NET (Ki-67 200x); (c) Grade 2 NET (Ki-67 200x); and (d) Grade 3 NET (Ki-67 200x) immunohistochemical Ki-67 staining

NET: neuroendocrine tumor

\section{Discussion}

Newly diagnosed NETs account for $\sim 0.5 \%$ of all malignancies. In a study conducted in the Netherlands, this type of tumor was mostly seen in the gastrointestinal system (62-67\%) and lungs (22-27\%) [15]. The estimated prevalence of gastroenteropancreatic NETs is $35 / 100,000$ people [16]. NETs comprise $2-5 \%$ of all pancreatic tumors and $25 \%$ of primary lung neoplasms [17]. In addition, $20 \%$ of NETs in the lung are small cell lung cancer, $3 \%$ are large cell neuroendocrine carcinoma, $1.8 \%$ are typical carcinoid tumors, and $0.2 \%$ are atypical carcinoid tumors $[17,18]$. Gastroenteropancreatic and lung NETs are graded according to histopathological features, mitosis rate, and the Ki-67 proliferation index [13,14]. NET cells have a uniform, round-oval small nuclei, inconspicuous nucleoli, and a chromatin pattern defined as salt and pepper in appearance [14]. NETs are tumors that immunohistochemically express neuroendocrine markers, such as chromogranin A and synaptophysin, and they characteristically show organoid, nesting, trabecular, or gyriform/serpentine growth patterns [19].

There are studies indicating that $12-22 \%$ of NETs have metastases during the first diagnosis [15]. In our study, there was a metastasis rate of $49.6 \%$, which presented mostly as liver, brain, and bone metastasis and was due to the higher proportion of NETs in the lungs and other organs and higher histological grades. Distant metastasis and local recurrence are the leading causes of death in patients with malignant neoplasia [20]. Inflammation contributes to the proliferation of malignant cells, angiogenesis, and metastasis in patients with tumors [21]. Inflammatory cells create a favorable environment for tumor growth early in the neoplastic process, facilitate genomic instability, and support angiogenesis. They then stimulate inflammatory mechanisms in tumor formation, such as selection-ligand interactions, matrix metalloprotein production, and chemokine functions, to support the neoplastic spread and metastasis [22]. Neutrophils can secrete various cytokines, such as granulocyte-macrophage colony-stimulating factor (GM-CSF), tumor necrosis factor-alpha (TNF-alpha), interleukin-1 (IL-1), and IL-8, to increase tumor formation, spread, and metastasis [23]. In addition, neutrophils can facilitate angiogenesis and metastasis by increasing tumor cell adhesion to the endothelium [24]. Neoplastic cells contain various membrane receptors that can bind directly to and activate platelets. Notably, platelets have been found to include factors that contribute to 
tumor growth, angiogenesis, and metastasis [25]. It has also been reported that systemic inflammation is associated with poor survival in various cancers [26].

A literature search yielded few studies comparing NLR and PLR with only histopathological parameters in NETs throughout the body. In our study, while there was a relationship between NETs in the lungs and other organs and NLR in terms of localization, we could not determine such relationships with PLR. However, because we have been unable to identify similar studies in the literature, further research on this subject may be beneficial. In various studies, the relationship between NLR and histological grade, lymphovascular invasion, and the Ki-67 proliferation index was determined in NETs. In these studies, the cut-off value for NLR was 2.4, 2.056, 2.2, and 2.31, respectively [9,10,20,27]. In another study, the NLR cut-off value was 2.8, but no relation was determined with the grade [28]. In our study, a high histological grade, a high Ki-67 proliferation index, high mitosis, and more lymphovascular invasion were determined in patients with cutoff values over 3.01 with NETs. Some studies, similar to ours, have found no relationship between NLR and perineural invasion [9]. In NETs, the relationship between PLR and grade was found in some studies with the cut-off values of 151.4 and 142, respectively [27,28]. In our study, no relation was found between PLR and histological grade, mitosis, the Ki-67 proliferation index, lymphovascular invasion, and perineural invasion. In pancreatic NETs, a relationship was found between patients whose NLR cut-off value was 2.4 and their TNM stage [9]. In NETs, the relationship between NLR and metastasis was determined in studies with a cutoff value of 2.2 and $2.8[20,28]$. In our study, distant metastases were detected in patients with an NLR cutoff value above 3.01. As in another study with a PLR cut-off value of 142, in our study, no relation was found between distant metastasis when the cut-off value was 134.4 [28]. The diagnosis of NETs can be made by biopsy from the primary organ or metastasis. Therefore, it is critical to investigate the factors related to metastases. Preoperative factors due to distant metastases in NETs are not well defined. Systemic inflammation markers such as NLR and PLR, which are easy to calculate, are advantageous in this respect.

In studies with NETs in the gastric system, pancreas, and lungs, the researchers mostly compared only clinical parameters and survival in these organs with NLR and/or PLR. Our study, which explores NETs in all systems and is based on pathological parameters, differs from those studies in this respect. Survival, lymph node metastasis, and distant metastases actually depend on the histopathological features of the tumor. Cut-off values are important for NLR and PLR. In some studies, cut-off values for NLR and PLR were determined empirically. In many studies, 5.0 was used as the cut-off value for NLR, while the cut-off value for PLR ranged from 150 to 300 [29]. In our study, the cut-off value was calculated with an ROC curve based on predicting distant metastasis for NLR and PLR. The best cut-off values for NLR and PLR were 3.01 and 134.4, respectively. The area under the curve was $75.6 \%$ and $63.1 \%$, respectively. These values show that NLR alone is superior to PLR as an independent predictive factor.

Our study had some limitations, including the use of a single center, the small number of cases, and the higher grade of tumors in the lungs and other organs. However, our review of NETs throughout the body and the use of histopathological parameters differentiate it from other studies.

\section{Conclusions}

In NETs, the diagnosis is commonly made by tru-cut, endoscopic, and bronchoscopic biopsies. The tumor area to be evaluated microscopically may be less than $2 \mathrm{~mm}^{2}$ in biopsies. Therefore, in small biopsies, the microscopic area where $\mathrm{Ki}-67$ and mitosis will be evaluated, which is important in determining the histological degree, may be insufficient. This is especially important in the differential diagnosis of grade 1 and grade 2 well-differentiated NETs. The evaluation of NLR in peripheral blood together with histopathological findings may be useful in follow-ups, especially for patients with a grade 1 or grade 2 NET diagnosis. In our study, a high histological grade, high mitosis, a high Ki-67 proliferation index, distant metastasis, and lymphovascular invasion were found in patients with an NLR cut-off value above 3.01. NLR, which is an easily accessible inflammatory marker, can be used as an independent predictive factor in NETs. However, we could not achieve similar results for PLR. NLR above certain values can be used for high-risk NET patients who need special treatment and close follow-up.

\section{Additional Information \\ Disclosures}

Human subjects: Consent was obtained or waived by all participants in this study. University of Health Sciences, Adana City Education and Research Hospital Ethics Committee issued approval 957. This study has been approved by the University of Health Sciences, Adana City Education and Research Hospital Ethics Committee. Animal subjects: All authors have confirmed that this study did not involve animal subjects or tissue. Conflicts of interest: In compliance with the ICMJE uniform disclosure form, all authors declare the following: Payment/services info: All authors have declared that no financial support was received from any organization for the submitted work. Financial relationships: All authors have declared that they have no financial relationships at present or within the previous three years with any organizations that might have an interest in the submitted work. Other relationships: All authors have declared that there are no other relationships or activities that could appear to have influenced the submitted work. 


\section{References}

1. Kim JY, Hong SM, Ro JY: Recent updates on grading and classification of neuroendocrine tumors . Ann Diagn Pathol. 2017, 29:11-6. 10.1016/j.anndiagpath.2017.04.005

2. Modlin IM, Oberg K, Chung DC, et al.: Gastroenteropancreatic neuroendocrine tumours. Lancet Oncol. 2008, 9:61-72. 10.1016/S1470-2045(07)70410-2

3. Kim J, Bae JS: Tumor-associated macrophages and neutrophils in tumor microenvironment . Mediators Inflamm. 2016, 2016:6058147. 10.1155/2016/6058147

4. Tomita M, Shimizu T, Ayabe T, Yonei A, Onitsuka T: Preoperative neutrophil to lymphocyte ratio as a prognostic predictor after curative resection for non-small cell lung cancer. Anticancer Res. 2011, 31:29958.

5. Kim EY, Lee JW, Yoo HM, Park CH, Song KY: The platelet-to-lymphocyte ratio versus neutrophil-tolymphocyte ratio: which is better as a prognostic factor in gastric cancer?. Ann Surg Oncol. 2015, 22:436370. 10.1245/s10434-015-4518-Z

6. Sun X, Liu X, Liu J, et al.: Preoperative neutrophil-to-lymphocyte ratio plus platelet-to-lymphocyte ratio in predicting survival for patients with stage I-II gastric cancer. Chin J Cancer. 2016, 35:57. 10.1186/s40880016-0122-2

7. Azab B, Shah N, Radbel J, et al.: Pretreatment neutrophil/lymphocyte ratio is superior to platelet/lymphocyte ratio as a predictor of long-term mortality in breast cancer patients. Med Oncol. 2013, 30:432. 10.1007/s12032-012-0432-4

8. Buergy D, Wenz F, Groden C, Brockmann MA: Tumor-platelet interaction in solid tumors . Int J Cancer. 2012, 130:2747-60. 10.1002/ijc.27441

9. Luo G, Liu C, Cheng H, et al.: Neutrophil-lymphocyte ratio predicts survival in pancreatic neuroendocrine tumors. Oncol Lett. 2017, 13:2454-8. 10.3892/ol.2017.5716

10. Tong Z, Liu L, Zheng Y, Jiang W, Zhao P, Fang W, Wang W: Predictive value of preoperative peripheral blood neutrophil/lymphocyte ratio for lymph node metastasis in patients of resectable pancreatic neuroendocrine tumors: a nomogram-based study. World J Surg Oncol. 2017, 15:108. 10.1186/s12957-017-1169-5

11. Salman T, Kazaz SN, Varol U, et al.: Prognostic value of the pretreatment neutrophil-to-lymphocyte ratio and platelet-to-lymphocyte ratio for patients with neuroendocrine tumors: an Izmir Oncology Group Study. Chemotherapy. 2016, 61:281-6. 10.1159/000445045

12. Pozza A, Pauletti B, Scarpa M, Ruffolo C, Bassi N, Massani M: Prognostic role of neutrophil-to-lymphocyte ratio and platelet-to-lymphocyte ratio in patients with midgut neuroendocrine tumors undergoing resective surgery. Int J Colorectal Dis. 2019, 34:1849-56. 10.1007/s00384-019-03356-5

13. WHO Classification of Tumours Editorial Board: WHO Classification of Tumours Series: Digestive System Tumours. International Agency for Research on Cancer, Lyon, France; 2019. https://publications.iarc.fr/Book-And-Report-Series/Who-Classification-Of-Tumours.

14. Travis WD, Brambilla E, Burke AP, Marx A, Nicholson AG: Introduction to The 2015 World Health Organization Classification of Tumors of the Lung, Pleura, Thymus, and Heart. J Thorac Oncol. 2015, 10:1240-2. 10.1097/JTO.0000000000000663

15. Taal BG, Visser O: Epidemiology of neuroendocrine tumours. Neuroendocrinology. 2004, 80:3-7. 10.1159/000080731

16. Niederle MB, Hackl M, Kaserer K, Niederle B: Gastroenteropancreatic neuroendocrine tumours: the current incidence and staging based on the WHO and European Neuroendocrine Tumour Society classification: an analysis based on prospectively collected parameters. Endocr Relat Cancer. 2010, 17:909-18. 10.1677/ERC10-0152

17. Oronsky B, Ma PC, Morgensztern D, Carter CA: Nothing but NET: a review of neuroendocrine tumors and carcinomas. Neoplasia. 2017, 19:991-1002. 10.1016/j.neo.2017.09.002

18. Hilal T: Current understanding and approach to well differentiated lung neuroendocrine tumors: an update on classification and management. Ther Adv Med Oncol. 2017, 9:189-99. 10.1177/1758834016678149

19. Klimstra DS, Modlin IR, Coppola D, Lloyd RV, Suster S: The pathologic classification of neuroendocrine tumors: a review of nomenclature, grading, and staging systems. Pancreas. 2010, 39:707-12. 10.1097/MPA.0b013e3181ec124e

20. Cao LL, Lu J, Lin JX, et al.: A novel predictive model based on preoperative blood neutrophil-to-lymphocyte ratio for survival prognosis in patients with gastric neuroendocrine neoplasms. Oncotarget. 2016, 7:4204558. 10.18632/oncotarget.9805

21. Colotta F, Allavena P, Sica A, Garlanda C, Mantovani A: Cancer-related inflammation, the seventh hallmark of cancer: links to genetic instability. Carcinogenesis. 2009, 30:1073-81. 10.1093/carcin/bgp127

22. Coussens LM, Werb Z: Inflammation and cancer. Nature. 2002, 420:860-7. 10.1038/nature01322

23. McColl SR, Paquin R, Ménard C, Beaulieu AD: Human neutrophils produce high levels of the interleukin 1 receptor antagonist in response to granulocyte/macrophage colony-stimulating factor and tumor necrosis factor alpha. J Exp Med. 1992, 176:593-8. 10.1084/jem.176.2.593

24. Liang W, Ferrara N: The complex role of neutrophils in tumor angiogenesis and metastasis . Cancer Immunol Res. 2016, 4:83-91. 10.1158/2326-6066.CIR-15-0313

25. Jain S, Harris J, Ware J: Platelets: linking hemostasis and cancer. Arterioscler Thromb Vasc Biol. 2010, 30:2362-7. 10.1161/ATVBAHA.110.207514

26. Li MX, Liu XM, Zhang XF, et al.: Prognostic role of neutrophil-to-lymphocyte ratio in colorectal cancer: a systematic review and meta-analysis. Int J Cancer. 2014, 134:2403-13. 10.1002/ijc.28536

27. Zhou B, Zhan C, Wu J, Liu J, Zhou J, Zheng S: Prognostic significance of preoperative neutrophil-tolymphocyte ratio in surgically resectable pancreatic neuroendocrine tumors. Med Sci Monit. 2017, 23:557488. 10.12659/msm.907182

28. Fan Y, Ma K, Niu W, Hu Y, Li E, Wu Y: Prognostic value of pretreatment prognostic nutritional index is superior to neutrophil to lymphocyte ratio for survival in patients with neuroendocrine tumors. Int J Clin Exp Pathol. 2017, 10:1719-28.

29. Zhang J, Zhang HY, Li J, Shao XY, Zhang CX: The elevated NLR, PLR and PLT may predict the prognosis of 


\section{Cureus}

patients with colorectal cancer: a systematic review and meta-analysis. Oncotarget. 2017, 8:68837-46. 10.18632/oncotarget.18575 